\title{
Alpha-Gal Allergy: a new threat to Appalachia
}

\author{
Makala Murphy, BS', Madison Griffis, MD', Adam M. Franks, MD², \\ Rebeka Franks, BA, CWR ${ }^{3}$, Colin M. Franks ${ }^{4}$, Gary Petty, MD²
}

ABSTRACT

Alpha-gal allergy, or mammalian meat allergy, is described as the development of IgE antibodies to the oligosaccharide galactose-a-1,3-galactose following a bite from the tick species Amblyomma americanum (Lone Star tick) or Dermacentor variabilis (Wood tick). Dermatologic or gastrointestinal symptoms are usually delayed by four to six hours after exposure, making the diagnosis difficult. Due to the use of mammalian proteins in many common medications, surgical equipment, and prosthesis, unexpected reactions can occur. In the United States, this pathology is predominately seen in the southeast but has been associated with other tick species on every continent except Antarctica. As the habitat for Amblyomma and Dermacentor continues to move further north due to changing patterns in deer population and weather, the incidence of alpha-gal syndrome has increased in the states outside its normal southeastern locale, especially in people with occupations and hobbies that require time outdoors in wooded areas.
Author affiliations are listed at the end of this article.

Correspondence to: Adam M. Franks, MD Marshall University Joan C. Edwards School of Medicine franks1@marshall.edu

\section{KEYWORDS}

Alpha-Gal, Allergies, Appalachia, Tick

\section{INTRODUCTION}

Alpha-gal allergy (AGA), also known as mammalian meat allergy, is a newly recognized pathology in which affected individuals can experience both new dermatologic (urticaria and angioedema) and gastrointestinal (nausea, diarrhea, and indigestion) symptoms due to antibodies against an oligosaccharide made by non-primate mammals. As symptoms follow a history of tick exposure, those with outdoor occupations and hobbies in wooded areas tend to be most at risk. The problem with the familiarity of this disease is multifactorial and includes recency of diagnosis, regionality, and delay in symptoms from exposure. Five cases of allergic reactions to dietary meat were initially discussed in the 1989 Georgia Allergy Society conference, ${ }^{1,2}$ but the pathophysiology was officially described in 2009 by Van Nunen. ${ }^{1,3,4}$ Cetuximab, a monoclonal antibody created in murine SP2/0 cells ${ }^{1,2,5}$ designed to treat unresectable metastatic colorectal cancer and head and neck squamous cell cancer, ${ }^{1-3,5,6-9}$ aided its discovery in the intervening twenty years. Immediate anaphylactic reactions to cetuximab were seen during investigational trials as early as $2004 .^{2}$ These reactions were largely confined to the southeastern United States (US), ${ }^{2}$ where they were seen 20 times as often as the remainder of the nation. ${ }^{1,6}$ In 2008, Chung discovered $20 \%$ of healthy controls from Tennessee had antibodies to portions of cetuximab, which in 2011 were confirmed to be related to the oligosaccharide alpha-gal. ${ }^{6}$

In addition to the southeastern US, AGA is seen from other tick species on every other continent except Antarctica (Table 1). The regionality of AGA in the US is primarily related to the distribution of Amblyomma americanum (Figure 1: Texas Lonestar tick) and, to a lesser extent, Dermacentor variabilis (Figure 2: Wood tick). 2,7,10-12 The deer population, on which the Lonestar tick feeds, is a primary factor restricting the range of this pathology, but this is changing. An overabundance of host species due to changing leash laws and hunting patterns has allowed this tick is spread to the upper Midwest and the east coast.,10 Changes in sub-freezing temperatures, humidity, and 


\section{Tick Species Associated with Alpha Gal Allergy}

Table\#1

\begin{tabular}{|l|l|}
\hline \multicolumn{1}{|c|}{ Continent } & \multicolumn{1}{c|}{ Tick } \\
\hline Africa & Amblyomma hebraeum* \\
\hline Asia & Haemaphysalis longicornis \\
\hline Australia & Ixodes holocyclus \\
\hline Europe & Ixodes ricinus \\
\hline North America & Ambylomma americanum (Lone Star tick) \\
\hline & Dermacentor variabillis (Wood Tick) \\
\hline & NOT Ixodes scapularis (Deer tick) \\
\hline South America & Ambylomma sculptum \\
\hline * suspected & \\
\hline
\end{tabular}

famotidine, intravenous fluids, and more diphenhydramine. Symptoms resolved in about an hour. While no lab work was ordered, chest radiograph and electrocardiogram were unremarkable.

Exam and vitals in the office four days later were unremarkable. A complete blood count, complete chemistry profile, and erythrocyte sedimentation rate were also normal. He denied new chemical, food, or environmental exposures. For his symptomatic days, he could only remember eating at Burger King ${ }^{\circledR}$ the evening of the most recent episode.
TABLE 1. Tick species on each continent that have been implicated in causing Alpha Gal Allergy.

ground temperature alter the questing and molting phases of the tick lifecycle, furthering the northward spread.,13

\section{CASE}

An 18-year-old Caucasian male with a history of seasonal allergies intermittently treated with cetirizine and a medication allergy to both amoxicillin and sulfa presented to the emergency department (ED) after two episodes of urticaria and pruritus over a one-month period. The initial episode began at midnight with urticaria on the trunk and arms, severe external pruritus, and mild pharyngeal pruritus. Within an hour, the urticaria resolved with diphenhydramine. He denied dyspnea, nausea, or swelling. The second episode occurred after midnight with a more severe "'burning"'" urticaria on the trunk and extremities. He also experienced angioedema of the face and hands, a cough, pharyngeal pruritus, mild dyspnea, chest tightness, and abdominal cramping with diarrhea. These symptoms were refractory to diphenhydramine at home. In the $E D$, he was treated with Solu-Medrol,
A. americanum, whole mounts of female (dorsal, left ventral right) Note "splotch" of crème color on posterior of scutum (dorsal surface) that gives this tick it's common name; the lone star tick. Specimens photographed in ethanol mounts

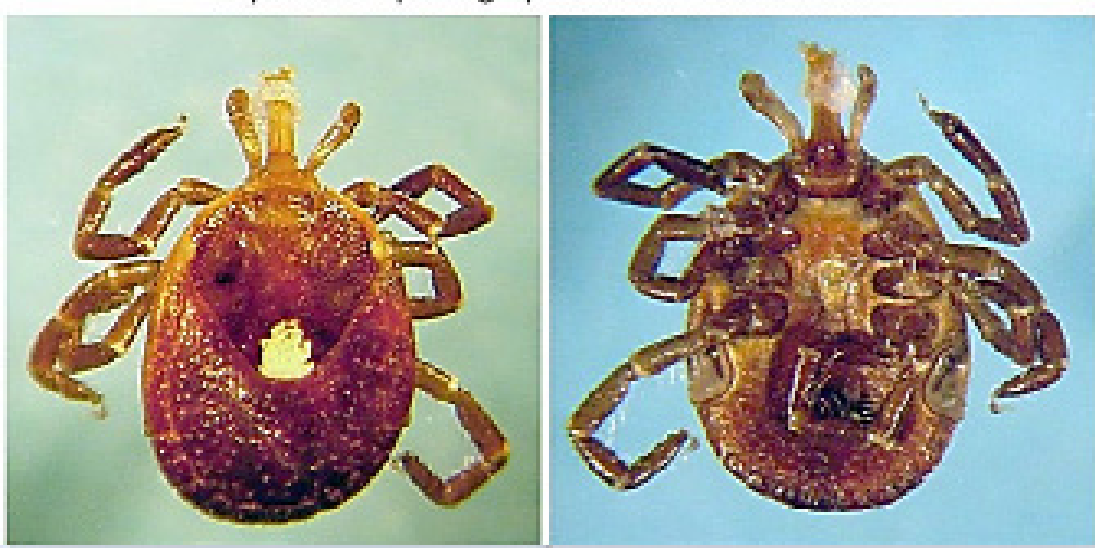

FIGURE 1: Amblyomma americanum (Texas Lonestar tick). Photos courtesy of James Joy, Digital Museum of Medical and Veterinary Acarology.

Dermacemor sp: temale (G); angorged famale (B) vertral views.
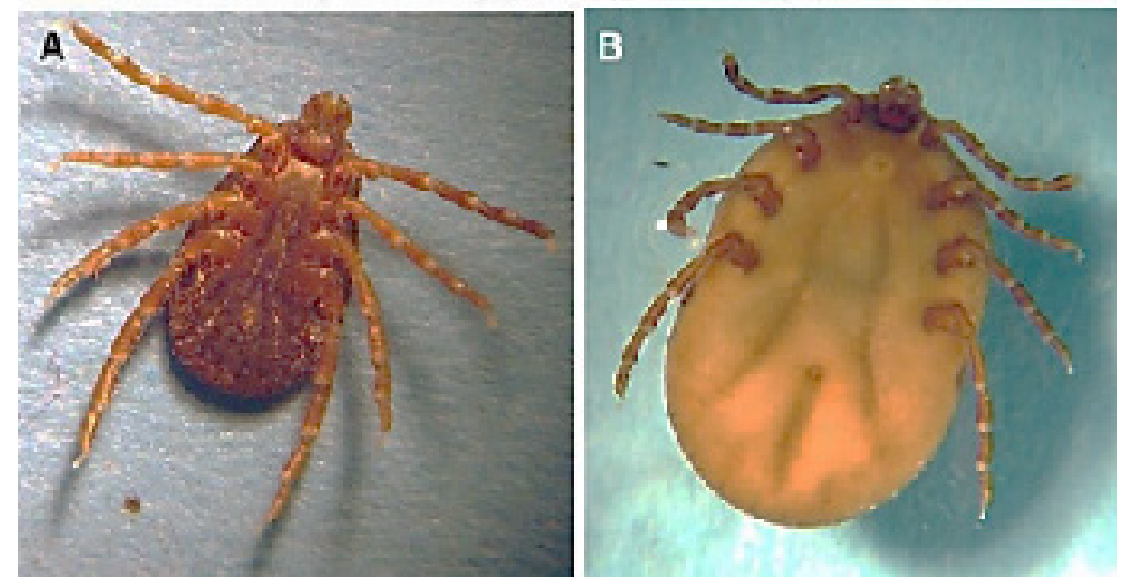

FIGURE 2: Dermacentor variabillis (Wood tick). Photos courtesy of James Joy, Digital Museum of Medical and Veterinary Acarology. 
Recent activities included hunting, fishing, and mowing lawns, which were associated with poison ivy, chiggers, and "pulling off a lot of ticks." Daily cetirizine was initiated, and he was given an EpiPen with instructions regarding its use. Due to worsening symptoms, he was scheduled to see allergy/ immunology.

In the month preceding his allergist appointment, he noted mild urticaria and "gagging" that occasionally led to emesis with cheese or milk consumption. These symptoms were not severe enough to require the use of his Epi-Pen. He did recall eating a hamburger without a severe reaction. The allergist ordered labs, and the patient was sent home with strict dietary instructions to avoid eating mammalian meats, limit milk and cheese, and monitor any reactions. The importance of tick avoidance measures was also discussed. Daily cetirizine and as-needed Epi-Pen were continued. Labs returned confirming AGA: complete blood count was normal, a tryptase level of 6.0 (normal 2.2 to 13.2), and a total IgE of 226.0 with an alpha-gal lgE of 46.9 (normal $<0.10$ ). Since the diagnosis, he has been able to reintroduce small amounts of cheese, but larger amounts have caused some gastrointestinal pain. He continues hunting and fishing but now uses insect repellent and is diligent about tick inspections after being outside.

\section{DISCUSSION}

\section{Pathophysiology}

Alpha-gal is a carbohydrate oligosaccharide, galactose-alpha-1,3-galactose, created by the alpha-1-3-galactosyltransferase gene. ${ }^{2,3}$ This gene is truncated in humans and catarrhine primates (Old World monkeys and apes) and produces a functionally inactive enzyme. ${ }^{2,3,7,11,14,15}$ Therefore, alpha-gal is seen as foreign to these species. Production in other mammals allows lgM/lgG sensitization across the gut from dietary consumption of meat and milk $^{3,6,14}$ as it survives heating, although freeze-drying and homogenization may diminish its presence. ${ }^{2,11}$ Alpha-gal lgM/lgG across the gut biome is thought to serve as a protective barrier to some strains of $E$. coli, Klebsiella, and Salmonella, as well as helminths, who share a commonality of surface epitopes with alpha-gal. 3,6,11,14

Despite this initial sensitization common to all humans, the development of a secondary hypersensitivity reaction is necessary to develop AGS. This occurs through the introduction of alpha-gal directly into the bloodstream through the prolonged feeding of a tick. In order for a tick to feed, it must disrupt the physical barrier of the epidermis, enter the dermis, and inhibit pro-inflammatory cytokines to overcome immunologic and hemostatic defenses. ${ }^{3}$ Interestingly, ticks also lack the enzyme to make alpha-gal, but through immunolocalization it is known to reside in their salivary secretory vesicles. Multiple theories have been postulated as to how alpha-gal is found in a species unable to construct it. Residual remains from a previous blood meal on non-primates are unlikely as alpha-gal has been seen in ticks naïve to those species. ${ }^{3,6,10}$ Coinfection with another organism, such as a rickettsial species, is possible but is still unproven. ${ }^{2,3,6}$ Cleavage of type $B$ blood is possible as it differs from alpha-gal epitopes by one fructose residue. ${ }^{3,8,10,16}$

Regardless, alpha-gal from tick saliva is required to trigger and augment AGA.3 A dual allergen exposure hypothesis requires both type 1 and type 2 hypersensitization (Figure 3).16 Alpha galspecific lgE from a type 1 hypersensitivity reaction seems necessary for AGA to occur, $1,3,4,6,10,17,18$ evidenced by increased levels of IgE after exposure that decreased over time with avoidance. ${ }^{4}$ These antibodies, produced in B lymphocyte memory cells, convert from producing alpha-gal specific lgM/ IgG after sensitization from the tick bite. ${ }^{1,3}$ After this conversion, symptomatic reactions occur as alpha-gal is absorbed from the gut and presented by basophils to the memory cells. These in turn, stimulate mast cells, ${ }^{1,2,19}$ whose numbers increase after subsequent infestations.3 The delay in symptom onset likely relates to the absorptive process as basophils typically elicit a reaction within 20 minutes. ${ }^{1-3,6,19}$ This multi-hour delay represents absorbing digested lipids as chylomicrons, repackaging, and transit delays. ${ }^{1,2}$ Factors affecting gut permeability and intestinal perfusion also affect symptom delay. ${ }^{3}$ Absorption is increased by alcohol consumption, ${ }^{1-3,6,16}$ high alpha-gal load (i.e., internal organs), and exercise. ${ }^{1,3,16}$ Fatty foods and non-steroidal anti-inflammatory 


\section{Potential Medical Complications Related to Alpha Gal Allergy}

\begin{tabular}{|c|c|c|}
\hline \multicolumn{3}{|c|}{ Effects on Heaith } \\
\hline & Cardionascular Disease & Increased atheromatous burden \\
\hline & Kounis Symdrome & Ischemic chest pain from allergic or immunologic causes \\
\hline \multicolumn{3}{|c|}{ Inoctive Compounds in Medications* } \\
\hline & Gelatin & $\begin{array}{l}\text { Found as an inactive component in gekaps, hemostatic agents, plasma } \\
\text { expanders, suppositories, and vaccines }\end{array}$ \\
\hline & Glycerin & $\begin{array}{l}\text { Found as an inactive component in some prparations of acetominophen, } \\
\text { anti-seizure medication, OTC cold \& GI preparations, prenatal vitamins, } \\
\text { progesterone, and vitamin supplements }\end{array}$ \\
\hline \multicolumn{3}{|c|}{ Lactic Acid } \\
\hline & Steric Acid & $\begin{array}{l}\text { Found as an inactive component in some preparations of acetominophen, } \\
\text { magnesium, opioids, and vitamin supplements }\end{array}$ \\
\hline \multicolumn{3}{|c|}{ Medications* } \\
\hline & Albumen & some is of Bovine origin \\
\hline & Antivenom & Equine and Ovine origin \\
\hline & Cetuximab & Murine origin \\
\hline & Collagen & Bovine and Equine origin \\
\hline & Hemostatic Agents & recombinent proteins from baby hamster kidney cell origin \\
\hline & Heparin & Porcine intestinal and Bovinf lung origin \\
\hline & Pancreatic Enzymes & Porcine pacrease origin \\
\hline \multicolumn{3}{|c|}{ Surgieal Issues } \\
\hline \multicolumn{3}{|c|}{$\begin{array}{r}\text { Biologic Mesh } \\
\end{array}$} \\
\hline & Biologic Suture & Catgut \\
\hline & Heart Valves & Bovine and porcine \\
\hline
\end{tabular}

TABLE 2. Medical conditions affected by Alpha Gal Allergy and commonly used medical products derived from animals.

medications slow the response. ${ }^{1,3,11,16}$

\section{DiAgNOSIS}

Delayed absorption is the primary impediment to the quick diagnosis of AGA. Studies show correct diagnoses occur in less than $10 \%$ of encounters. ${ }^{20}$ A suspicion for a meat allergy aids a good history which included: a previous history of asymptomatic meat consumption, dermatologic symptoms 2-6 hours after eating meat, absence of symptoms in a meat-restricted diet, and a history of tick exposure. ${ }^{1,2,6}$ Diagnostic testing confirms historical information with a positive serum alpha-gal lgE ratio of $>/=2 \mathrm{IU} / \mathrm{mL}$ or a ratio $>2 \%$ (normal $</=1 \%$ ) of the total serum IgE.3,11 False positives can occur in parasitized patients, 5 but levels tend to correlate with clinical disease. 11 Other tests are intradermal skin prick tests using milk, beef, or pork with turkey, chicken, or fish control, ${ }^{1-3}$ as well as basophil activation tests using cetuximab. ${ }^{11}$ However, these tests should be used in conjunction with IgE levels due to highly variable sensitivity. ${ }^{3}$ Tryptase levels demonstrate an allergic reaction has occurred. ${ }^{17}$ The gold standard of an oral food challenge, either with a 150-gram pork load or incremental increases every 15 minutes, is not recommended because of the delay in symptoms.

Treatment/Prognosis

There is no definitive cure for AGA. The treatment cornerstone is avoidance of red meat, $1,6,9,11,16$ although evidence is insufficient to recommend avoiding dairy products. ${ }^{1}$ Patients should also avoid further tick or other ectoparasites (chigger) ${ }^{1,18}$ bites by wearing proper clothing, utilizing protective sprays, and suppressing host-seeking ticks.,17 Evidence suggests avoidance of further tick bites may decrease alpha-gal lgE and symptoms over time, but symptoms can return with new exposures. ${ }^{3,6,11}$ 
Equally important in the treatment of AGA is identifying potential patient risks, including medical risks (Table 2 ) and treating reactions. Thorough medical record documentation, a written action plan, and medical alert bracelets may be helpful., ${ }^{6,11}$ Plans should include early identification and management of symptoms. Should symptoms occur, histamine blockers, steroids, albuterol, epinephrine, oxygen, intravenous fluids, or simply observation/supportive care may all be included depending on severity. ${ }^{6,11}$ Patients should be educated about the rarity of AGA as they may experience hardship and frustration when medical providers lack sufficient knowledge regarding this process. Furthermore, patients must feel empowered for self-advocacy to drive their medical care if needed. ${ }^{18}$

\section{CONCLUSION}

AGA is a relatively new, incompletely explored pathology. Delay in symptomatology following mammalian meat consumption makes diagnosis difficult and requires clinician suspicion to diagnose quickly. As behavioral and environmental factors are allowing its spread outside of the typically confined area in the southeastern US, providers unfamiliar with the pathology are encountering it. Physician awareness is essential to help patients manage the associated dietary and medical issues.

\section{AUTHOR AFFILIATIONS}

1. Marshall University Joan C. Edwards School of Medicine, Huntington, West Virginia

2. Marshall University Joan C. Edwards School of Medicine, Department of Family and Community Health, Huntington, West Virginia

3. Heritage Farms Foundation Smithsonian Affiliate, Huntington, West Virginia

4. Marshall University, Huntington, West Virginia

\section{REFERENCES}

1. Platts-Mills TAE, Li RC, Keshavarz B, Smith AR, Wilson JM. Diagnosis and Management of Patients with the a-Gal Syndrome. J Allergy Clin
Immunol Pract. 2020;8(1):15-23.e1. doi:10.1016/j. jaip.2019.09.017

2. Steinke JW, Platts-Mills TA, Commins SP. The alpha-gal story: lessons learned from connecting the dots. J Allergy Clin Immunol. 2015;135(3):589597. doi:10.1016/j.jaci.2014.12.1947

3. Cabezas-Cruz A, Hodžić $A$, Román-Carrasco $P$, et al. Environmental and Molecular Drivers of the a-Gal Syndrome. Front Immunol. 2019;10:1210. Published 2019 May 31. doi:10.3389/ fimmu.2019.01210

4. Lied GA, Lund KB, Storaas T. Intraoperative anaphylaxis to gelatin-based hemostatic agents: a case report. J Asthma Allergy. 2019;12:163-167. Published 2019 Jun 18. doi:10.2147/JAA.S202784

5. Michel S, Scherer K, Heijnen IA, Bircher AJ. Skin prick test and basophil reactivity to cetuximab in patients with IgE to alpha-gal and allergy to red meat. Allergy. 2014;69(3):403-405. doi:10.1111/ all.12344

6. Dunkman WJ, Rycek W, Manning MW. What Does a Red Meat Allergy Have to Do With Anesthesia? Perioperative Management of Alpha-Gal Syndrome. Anesth Analg. 2019;129(5):1242-1248. doi:10.1213/ANE.0000000000003460

7. Chinuki Y, Morita E. Alpha-Gal-containing biologics and anaphylaxis. Allergol Int. 2019;68(3):296-300. doi:10.1016/j.alit.2019.04.001

8. Tjernberg I, Hamsten C, Apostolovic D, van Hage $M$. IgE reactivity to a-Gal in relation to Lyme borreliosis. PLoS One. 2017;12(9):e0185723. Published 2017 Sep 27. doi:10.1371/journal. pone. 0185723

9. Caglayan-Sozmen S, Santoro A, Cipriani F, Mastrorilli C, Ricci G, Caffarelli C. Hazardous Medications in Children with Egg, Red Meat, Gelatin, Fish, and Cow's Milk Allergy. Medicina (Kaunas). 2019;55(8):501. Published 2019 Aug 19. doi:10.3390/medicina55080501

10. Crispell G, Commins SP, Archer-Hartman SA, et al. Discovery of Alpha-Gal-Containing Antigens in North American Tick Species Believed to Induce Red Meat Allergy. Front Immunol. 2019;10:1056. Published 2019 May 17. doi:10.3389/ fimmu.2019.01056

11. Mabelane T, Ogunbanjo GA. Ingestion of mammalian meat and alpha-gal allergy: Clinical relevance in primary care. Afr J Prim Health Care Fam Med. 2019;11(1):e1-e5. Published 2019 Apr 29. doi:10.4102/phcfm.v11i1.1901 
12. Hamsten C, Tran TAT, Starkhammar M, et al. Red meat allergy in Sweden: association with tick sensitization and B-negative blood groups. J Allergy Clin Immunol. 2013;132(6):1431-1434. doi:10.1016/j.jaci.2013.07.050

13. Linske MA, Williams SC, Stafford KC 3rd, et al. Determining Effects of Winter Weather Conditions on Adult Amblyomma americanum (Acari: Ixodidae) Survival in Connecticut and Maine, USA. Insects. 2019;11(1):13. Published 2019 Dec 21. doi:10.3390/insects 11010013

14. Hodžić $A$, Mateos-Hernández $L$, Leschnik $M$, et al. Tick Bites Induce Anti-a-Gal Antibodies in Dogs. Vaccines (Basel). 2019;7(3):114. Published 2019 Sep 15. doi:10.3390/vaccines7030114

15. Stone CA Jr, Hemler JA, Commins SP, et al. Anaphylaxis after zoster vaccine: Implicating alpha-gal allergy as a possible mechanism. J Allergy Clin Immunol. 2017;139(5):1710-1713.e2. doi:10.1016/j.jaci.2016.10.037

16. Wilson JM, Schuyler AJ, Workman L, et al. Investigation into the a-Gal Syndrome: Characteristics of 261 Children and Adults Reporting Red Meat Allergy. J Allergy Clin Immunol Pract. 2019;7(7):2348-2358.e4. doi:10.1016/j.jaip.2019.03.031

17. Radwan SS, Gill G, Ghazzal A, Malik A, Barnett

C. Plaque Rupture-Induced Myocardial Infarction and Mechanical Circulatory Support in Alpha-Gal Allergy. Case Rep Cardiol. 2020;2020:5282843. Published 2020 Feb 17. doi:10.1155/2020/5282843

18. Stoltz LP, Cristiano LM, Dowling APG, Wilson $J M$, Platts-Mills TAE, Traister RS. Could chiggers be contributing to the prevalence of galactose-alpha-1,3-galactose sensitization and mammalian meat allergy?. J Allergy Clin Immunol Pract. 2019;7(2):664-666. doi:10.1016/j. jaip.2018.07.014

19. Apostolovic D, Mihailovic J, Commins SP, et al. Allergenomics of the tick Ixodes ricinus reveals important a-Gal-carrying lgE-binding proteins in red meat allergy. Allergy. 2020;75(1):217-220. doi:10.1111/all.13978

20. Flaherty MG, Kaplan SJ, Jerath MR. Diagnosis of Life-Threatening Alpha-Gal Food Allergy Appears to Be Patient Driven. J Prim Care Community Health. 2017;8(4):345-348.

doi:10.1177/2150131917705714 